\title{
BICAVAL AND STANDARD TECHNIQUES IN ORTHOTOPIC HEART TRANSPLANTATION: MEDIUM-TERM EXPERIENCE IN CARDIAC PERFORMANCE AND SURVIVAL
}

Tarek Aziz, FRCS

Malcolm Burgess, MRCPa

Richard Khafagy, $\mathrm{MB}, \mathrm{ChB}^{\mathrm{a}}$

Alison Wynn Hann, $\mathrm{PhD}^{\mathrm{b}}$

Colin Campbell, FRCS ${ }^{\mathrm{a}}$

Ali Rahman, FRCS ${ }^{\mathrm{a}}$

Abdul Deiraniya, FRCS ${ }^{\text {a }}$

Nizar Yonan, FRCS
Objective: The aim of this study was to compare the medium-term results of right heart pressures, tricuspid valve dysfunction, overall cardiac performance, and survival between the bicaval and standard techniques. Method: Between 1991 and 1997, 201 heart transplantations were performed in our center. Right heart catheterization was performed up to 12 months after transplantation. Echocardiography was used to assess left ventricular and tricuspid valve function. Result: The standard technique was used in $\mathbf{1 0 5}$ cases, and the bicaval technique was used in 96 cases. There was no difference in the age, preoperative parameters, pulmonary hemodynamics, or ischemic time between the 2 groups. Right atrial pressure $(4.3 \pm 4.0 \mathrm{~mm} \mathrm{Hg}$ for the bicaval vs $10.9 \pm 4.8 \mathrm{~mm}$ Hg for standard technique) and mean pulmonary artery pressure (17.5 $\pm 5.3 \mathrm{~mm} \mathbf{H g}$ and $22.5 \pm 5.2 \mathrm{~mm} \mathrm{Hg}$, respectively) were lower for the bicaval recipients up to 12 months after the operation $(P=.001$ and .01 , respectively). Left ventricular ejection fraction was higher for the recipients of the bicaval technique up to the most recent measurement $(P=$ .005). The prevalence of moderate or severe tricuspid regurgitation was higher in the recipients of the standard technique up to the most recent measurement $(28 \%$ vs $7 \% ; P=.02)$. The actuarial survival at 1,3 , and 5 years was $74 \%, 70 \%$, and $62 \%$ for the recipients of the standard technique versus $87 \%, 82 \%$, and $81 \%$ for the recipients of the bicaval technique $(P<.03,<.04$, and $<.02$, respectively $)$. Conclusion: The bicaval technique maintains good left ventricular function, lower incidence and severity of tricuspid valve dysfunction, and improved survival compared with the standard technique. (J Thorac Cardiovasc Surg 1999;118: 115-22)
0 rthotopic heart transplantation is a well-established method for treatment of end-stage heart failure. Most orthotopic heart transplantation procedures have been performed according to the technique described by Sarsam and colleagues ${ }^{1}$ and by Lower, Stofer, and Shumway. $^{2}$ The bicaval technique ${ }^{3}$ with a small left atrial cuff and cavoatrial anastomoses was introduced

From the Cardiac Transplant Unit, Wythenshawe Hospital, ${ }^{\mathrm{a}}$ and the University Department of Statistics, ${ }^{\mathrm{b}}$ Manchester University, Manchester, United Kingdom.

Presented at the Twelfth European Association of Cardiothoracic Surgery, Brussels, Belgium, 1998.

Received for publication July 30, 1998; revisions requested Sept 9, 1998; revisions received March 5, 1999; accepted for publication March 8, 1999.

Address for reprints: T. M. Aziz, FRCS, Cardiac Transplant Unit, Wythenshawe Hospital, Southmoor Road, Manchester, UK, M23 9LT.

Copyright (C) 1999 by Mosby, Inc.

$0022-5223 / 99 \$ 8.00+0 \quad \mathbf{1 2 / 1 / 9 8 4 2 6}$ into the clinical practice of orthotopic heart transplantation in 1991.

The theoretic advantage of this alternative technique is to retain normal-shaped atria, which may preserve atrial contractility and sinus node function and may maintain the atrioventricular valve competence. ${ }^{4,5}$

The influence of the bicaval technique on hemodynamics, right atrial (RA) contractility, and right ventricular dimension during the early postoperative period has been previously described. ${ }^{6-8}$ This study was designed to compare the medium-term difference between the standard and the bicaval techniques for orthotopic heart transplantation and to demonstrate any long-term advantages of RA preservation on right-sided output that optimizes right-sided cardiac pressure. We also compared the left ventricular performance and actuarial survival after the introduction of the bicaval technique into the clinical practice of orthotopic heart transplantation. 
Table I. Pretransplantation pathologic condition in 201 heart transplant recipients

\begin{tabular}{lcc}
\hline & \multicolumn{2}{c}{ No. of patients } \\
\cline { 2 - 3 } Pretransplantation & $\begin{array}{c}\text { Standard } \\
\text { technique } \\
(n=105)\end{array}$ & $\begin{array}{c}\text { Bicaval } \\
\text { technique } \\
\text { pathologic condition }\end{array}$ \\
\hline Ischemic heart disease & 59 & $63)$ \\
Cardiomyopathy & 33 & 30 \\
Valve disease & 5 & 8 \\
Other heart conditions & 2 & 1 \\
\hline
\end{tabular}

\section{Methods}

Patient characteristics. The patient population consisted of 201 heart transplant recipients (mean age, $49 \pm 10$ years; range, 12-62 years); 39 women and 162 men who underwent orthotopic heart transplantation between 1991 and 1997 at Wythenshawe Hospital, Manchester, United Kingdom. The preoperative pathologic condition of the whole study group is listed in Table I.

Patients were allocated on an alternate basis to undergo operation by either the bicaval Wythenshawe ${ }^{1}$ technique $(\mathrm{n}=$ 96 patients) or the standard technique of Lower, ${ }^{2}$ Shumway, ${ }^{3}$ and their colleagues ( $\mathrm{n}=105$ patients). For a temporary period of 6 months (December 1993 through June 1994), the operative technique was determined by coin tossing. Patients undergoing retransplantation, patients receiving domino hearts (very short superior vena cava and difficult to perform superior vena cava anastomosis), and patients in whom performance of bicaval anastomosis was technically difficult (high diaphragm or other chest cavity anomalies) were excluded from the study. There was no difference in mean preoperative pulmonary hemodynamics, age, ischemic time, and postoperative incidence of rejection between the 2 groups. Follow-up was completed on April 1998 or at the time of the recipient's death, thus giving a minimum followup of 12 months for all surviving patients.

Postoperative management. All patients received isoproterenol (INN: isoprenaline) intravenously for a minimum of 3 days as part of our routine postoperative protocol. Other cardiac inotropic or mechanical supports (intra-aortic balloon pump or right-sided ventricular assist device) were used whenever clinically indicated in both groups. Triple-drug immunosuppression with cyclosporine (INN: ciclosporin), azathioprine, and corticosteroids was used in all patients in addition to an initial 3-day dose of cytolytic induction therapy. Surveillance endomyocardial biopsies (EMBs) were performed on a scheduled basis (weekly for the first month; every 2 weeks for the next 2 months; monthly up to 6 months; at 9 , 12 , and 18 months after the transplantation; and annually thereafter). If rejection was suspected on clinical grounds in the interim, supplementary biopsies were performed accordingly.

Surgical techniques. The standard technique was performed as described by Lower, ${ }^{2}$ Shumway, ${ }^{3}$ and their colleagues. The bicaval technique was performed as previously described by Sarsam and colleagues. ${ }^{1}$ In brief, the right atrium of the recipient is excised, leaving behind a 2- to $3-\mathrm{cm}$ cuff around each cava (cavoatrial cuff). The left atrial incision is carried to the base of the left atrial appendage, which is removed leaving a small margin of the atrial cuff around 4 pulmonary veins. The donor hearts are excised with an intact right atrium and long cava. Donor hearts are arrested with cold St Thomas' Hospital cardioplegic solution and stored in $4^{\circ} \mathrm{C}$ cold saline solution. The donor left atrium is sutured to the recipient left atrium in the usual fashion before the inferior vena cava and the superior vena cava are sutured to the recipient cavoatrial cuff. The aorta and pulmonary arteries are sutured in the usual way.

Endomyocardial biopsy and right heart catheterization. The policy at our institution is for EMBs to be performed with a Stanford-Caves bioptome, usually through a percutaneous right internal jugular approach. Right heart catheterization is performed with a Swan-Ganz catheter (Baxter Healthcare Corp, Edwards Division, Santa Ana, Calif) or a multipurpose Cordis $7 \mathrm{~F}$ vascular catheter (Cordis, Miami, Fla) connected to an AE 840 pressure transducer (Mikro Elektronik K A/S) during each biopsy up to 12 months after the transplantation, when previous EMB reports indicate cellular rejection of grade 2 or more or whenever there was a clinical suggestion of rejection. For the purposes of the present study, right heart pressure assessment was performed according to our usual protocol. At different study periods (except the 4-week periods), right-sided cardiac pressure measurements were made, provided there had been no histologic evidence of rejection within the preceding 3 months. Hemodynamic assessment was only considered in the absence of rejection (ie, International Society for Heart and Lung Transplantation grade 0). All patients were in sinus rhythm without cardiac pacing at the time of assessment.

Echocardiography. Comprehensive transthoracic echocardiography (with color flow mapping) was performed in each patient at the same time as EMBs up to the end of the first year after the transplantation, and then every 6 months or when clinically indicated. The examination was performed with patients in the left lateral decubitus position in quiet respiration after 5 minutes of rest with a machine (Sonos 2000/2500; Hewlett-Packard Company, Andover, Mass) equipped with a $2.5 \mathrm{MHz}$ transducer. An experienced echocardiographer who was unaware of the surgical technique as part of the routine echocardiographic evaluation performed each study. The assessment of left ventricular function was on a semiquantitative basis with visual estimation of ejection fraction after imaging of the left ventricular myocardium in the parasternal long-axis and short-axis views and the apical 4-chamber and long-axis views. The tricuspid valve was interrogated in the parasternal short-axis and apical 4-chamber views. Color flow Doppler examinations were performed with as narrow a sector angle as possible to maximize the imaging frame rate, and gain was adjusted from a high level downward to a point where aliasing just disappeared. The assessment of tricuspid valve dysfunction was semiquantitative and reliant on a combination of the continu- 
Table II. Preoperative parameters in the recipients of the standard and bicaval techniques before heart transplantation

\begin{tabular}{lccc}
\hline & $\begin{array}{c}\text { Standard } \\
\text { technique }\end{array}$ & $\begin{array}{c}\text { Bicaval } \\
\text { technique }\end{array}$ & P value \\
\hline Age (y) & $49 \pm 9.9$ & $47 \pm 11.2$ & .7 \\
Sex (F/M) & $17 / 105$ & $12 / 96$ & .06 \\
Before transplantation & & & \\
$\quad$ IABP (n) & $11 / 105$ & $12 / 96$ & .08 \\
$\quad$ Inotropic drugs (n) & $18 / 105$ & $20 / 96$ & .6 \\
$\quad$ Ventilation (n) & $1 / 105$ & $2 / 96$ & .8 \\
Previous heart surgery (n) & $21 / 105$ & $18 / 96$ & .7 \\
EF (\%) & $15.1 \pm 4.9$ & $15.9 \pm 5.1$ & .6 \\
RA pressure & $14.0 \pm 6.0$ & $15.0 \pm 8.0$ & .9 \\
$\quad$ (mm Hg \pm mean) & & & \\
PA pressure & $33.0 \pm 8.2$ & $33.0 \pm 9.8$ & .8 \\
$\quad$ (mm Hg mean) & & & \\
TPG (mm Hg) & $8.1 \pm 3.8$ & $8.0 \pm 3.7$ & .9 \\
PVR (Wood units) & $2.5 \pm 1.1$ & $2.6 \pm 1.3$ & .9 \\
PCWP (mm Hg) & $22.6 \pm 9.2$ & $24.4 \pm 9.1$ & .8 \\
Ischemic time (min) & $179.0 \pm 39.0$ & $182.0 \pm 40.0$ & .8 \\
Implant time (min) & $58.0 \pm 9.5$ & $60.0 \pm 10.0$ & .8 \\
Donor age (y) & $33.6 \pm 8.7$ & $32.1 \pm 10.5$ & .8 \\
\hline
\end{tabular}

$I A B P$, Intra-aortic balloon pump; $E F \%$, ejection fraction; $T P G$, transpulmonary gradient; $P V R$, pulmonary vascular resistence; $P C W P$, pulmonary capillary wedge pressure.

ous wave and color-flow Doppler findings. In the former, tricuspid valve dysfunction was considered present if blue, green, or mosaic signals originated from the atrial aspect of the tricuspid valve during systole. The major criterion for the severity of regurgitation was qualitative assessment according to the ratio of regurgitation jet area, as previously described. In general, a regurgitation jet area/atrial area of less than $10 \%$ was considered trivial, $10 \%$ to $25 \%$ was considered mild, $25 \%$ to $50 \%$ was considered moderate, and more than $50 \%$ was considered severe. Echocardiographic assessment was performed in the absence of rejection after the same policy of hemodynamics assessment. All patients were in sinus rhythm without cardiac pacing at the time of echocardiography.

Data analysis. Data are expressed as mean \pm standard deviation. Data between the 2 groups were compared with the Mann-Whitney test, $t$ test, or $\chi^{2}$ where applicable. Survival was calculated by Kaplan-Meier analysis. In view of possible changes in transplantation, all investigations were divided into those performed in 2 eras of transplantation practice. Era 1 was from April 1991 to April 1994 (38 recipients with the standard technique and 41 recipients with the bicaval technique) and era 2 from April 1994 to April 1997 (67 recipients with the standard and 55 recipients with the bicaval technique).

\section{Results}

Early postoperative course. The operative mortality rate was $16 \%$ (18/105 cases) for the standard group
Table III. Right heart catheter measurements in recipients of the standard and bicaval techniques after transplantation

\begin{tabular}{lccc}
\hline & $\begin{array}{c}\text { Standard } \\
\text { technique } \\
(n=105)\end{array}$ & $\begin{array}{c}\text { Bicaval } \\
\text { technique } \\
(n=96)\end{array}$ & P value \\
\hline Parameters & $71.0 \pm 14$ & $68.0 \pm 16$ & .6 \\
\hline $\begin{array}{l}\text { Aortic pressure } \\
\text { (mm Hg) }\end{array}$ & $2.5 \pm 0.5$ & $3.8 \pm 0.9$ & .02 \\
Cardiac index & $13.7 \pm 3.2$ & $7.9 \pm 2.9$ & .01 \\
RA pressure & & & \\
$\quad$ postop; mm Hg) & $11.7 \pm 2.6$ & $5.7 \pm 4.6$ & .001 \\
RA 4 (mm Hg) & $10.9 \pm 4.8$ & $4.3 \pm 4.0$ & .001 \\
RA 12 (mm Hg) & $36.0 \pm 7.9$ & $28.0 \pm 6.2$ & .001 \\
RV 4 (mm Hg) & $32.0 \pm 5.9$ & $27.0 \pm 6.9$ & .02 \\
RV 12 (mm Hg) & $27.9 \pm 5.6$ & $22.1 \pm 5.3$ & .002 \\
Mean PA* & & & \\
$\quad$ postop; $\mathrm{mm} \mathrm{Hg})$ & $23.6 \pm 4.2$ & $17.3 \pm 4.8$ & .001 \\
Mean PA 4 $(\mathrm{mm} \mathrm{Hg})$ & $22.5 \pm 5.2$ & $17.5 \pm 5.3$ & .01 \\
Mean PA 12 $(\mathrm{mm} \mathrm{Hg})$ & &
\end{tabular}

$R A 4$, RA pressure at 4 weeks after transplantation; $R A 12$, RA pressure at 12 months after transplantation; $R V 4$, right ventricular pressure at 4 weeks; $R V$ 12 , systolic right ventricular pressure 12 months after operation.

* Mean PA pressure before surgery.

Mean PA pressure 4 weeks after transplantation.

${ }^{*}$ Mean PA pressure 12 months after transplantation.

versus $6.2 \%$ (6/96 patients; $P=.03$ ) for the recipients of the bicaval technique. Ischemic time, donor age, and implantation times were similar in the 2 groups (Table II). Hemodynamic parameters recorded during the early postoperative period are summarized in Table III. There was no significant difference in the mean aortic pressure between the 2 groups, but the cardiac index was significantly higher for the recipients of the bicaval technique. The mean RA blood pressure and mean pulmonary artery (PA) pressures were significantly lower in the recipients of the bicaval technique (Table III). Pulmonary wedge pressure was also lower but not significantly different for the bicaval group during the early postoperative period $(P=.1)$. The mean ventilation time in hours and the hours of intensive care stay were shorter for the recipients of the bicaval technique compared with the recipients of the standard technique $(P=.001$ and $P=.01$, respectively). Analysis of the results per era revealed that the cardiac index $(\mathrm{CI})$ for the recipients of the bicaval technique $(\mathrm{CI}, 3.7 \pm 1.1$ $\mathrm{L} / \mathrm{m}^{2}$ for era 1 ; CI, $3.87 \pm 0.5 \mathrm{~L} / \mathrm{m}^{2}$ for era 2) was significantly higher than that of recipients of the standard technique during the same periods $\left(\mathrm{CI}, 2.56 \pm 0.2 \mathrm{~L} / \mathrm{m}^{2}\right.$ for era $1 ; \mathrm{CI}, 2.48 \pm 0.6$ for era $2 ; P=.02$ and $P=.03$, respectively, for the difference between techniques during each era). RA and mean PA pressures were significantly lower in the recipients of the bicaval technique 


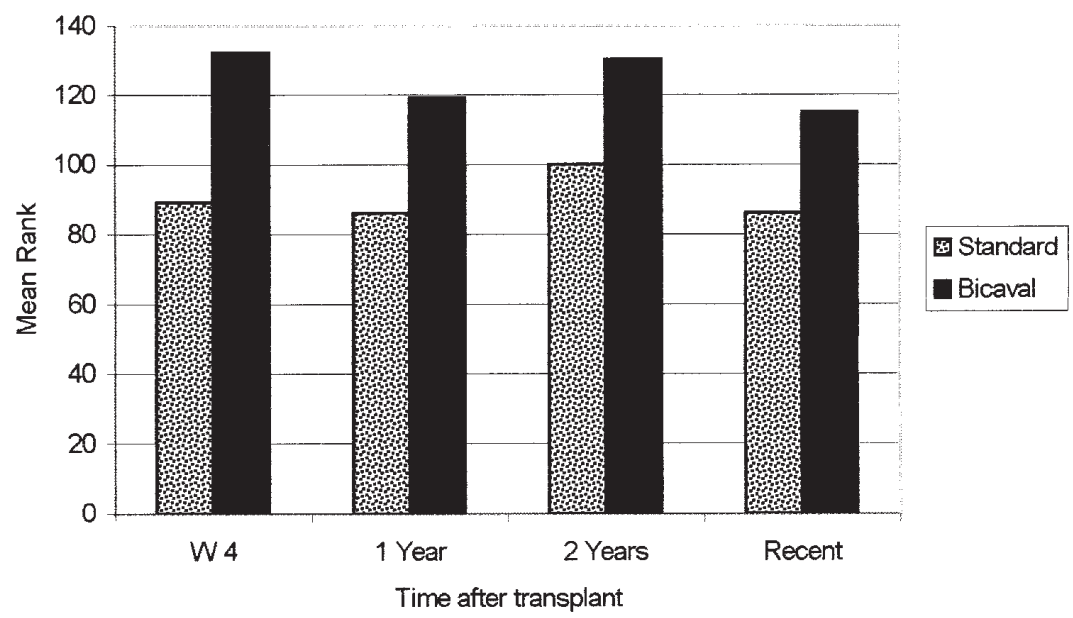

Fig 1. Mann-Whitney-Wilcoxon rank to compare the left ventricular ejection fraction between the recipients of the bicaval and standard techniques at different intervals.

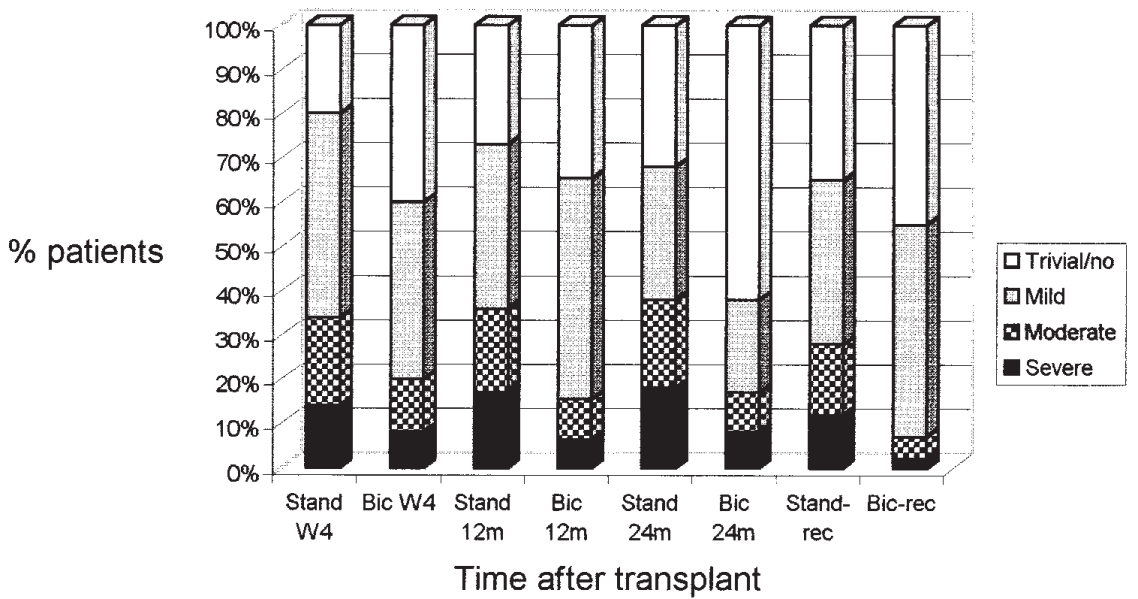

Fig 2. Prevalence and severity of tricuspid valve dysfunction after heart transplantation in recipients of the standard and bicaval techniques.

during era 1 (RA, $7.8 \pm 2.8 \mathrm{~mm} \mathrm{Hg}$; PA, $22.6 \pm 4.4 \mathrm{~mm}$ $\mathrm{Hg}$ ) compared with the recipients of the standard technique that was performed during the same era (RA, $12.8 \pm 3.9 \mathrm{~mm} \mathrm{Hg} ; \mathrm{PA}, 27.5 \pm 5.8 \mathrm{~mm} \mathrm{Hg} ; P=.01$ and .001 , respectively). During era 2 , the right heart pressures were also lower for the recipients of the bicaval technique (RA, $7.9 \pm 3.1 \mathrm{~mm} \mathrm{Hg}$; PA, $21.7 \pm 5.6 \mathrm{~mm}$ $\mathrm{Hg}$ ) than for the recipients of the standard technique performed during the same era (RA, $13.9 \pm 2.9 \mathrm{~mm} \mathrm{Hg}$; PA, $28.0 \pm 5.5 \mathrm{~mm} \mathrm{Hg} ; P=.01$ and .002 , respectively). Average ventilation time and intensive therapy unit (ITU) stay were shorter for patients in era 2 with both techniques but still significantly longer for the recipients of the standard technique. During era 1 , mean ven- tilation time was $69 \pm 45$ hours and mean ITU stay was $84 \pm 60$ hours for the recipients of the bicaval technique compared with $89 \pm 72$ hours and $134 \pm 48$ hours for the recipients of the standard technique $(P=.002$ and .007 , respectively). During era 2 , mean ventilation time was $58 \pm 42$ hours, and mean ITU stay was $79 \pm 45$ hours for the recipients of the bicaval technique compared with mean ventilation time of $74 \pm 66$ hours and ITU stay of $107 \pm 60$ hours for the recipients of the standard technique ( $P=.001$ and .02 , respectively).

Four-week period. Four weeks after transplantation, the right-sided filling pressures (RA, right ventricular, and mean pulmonary) were higher for the standard group (Table III). The pulmonary capillary wedge pres- 


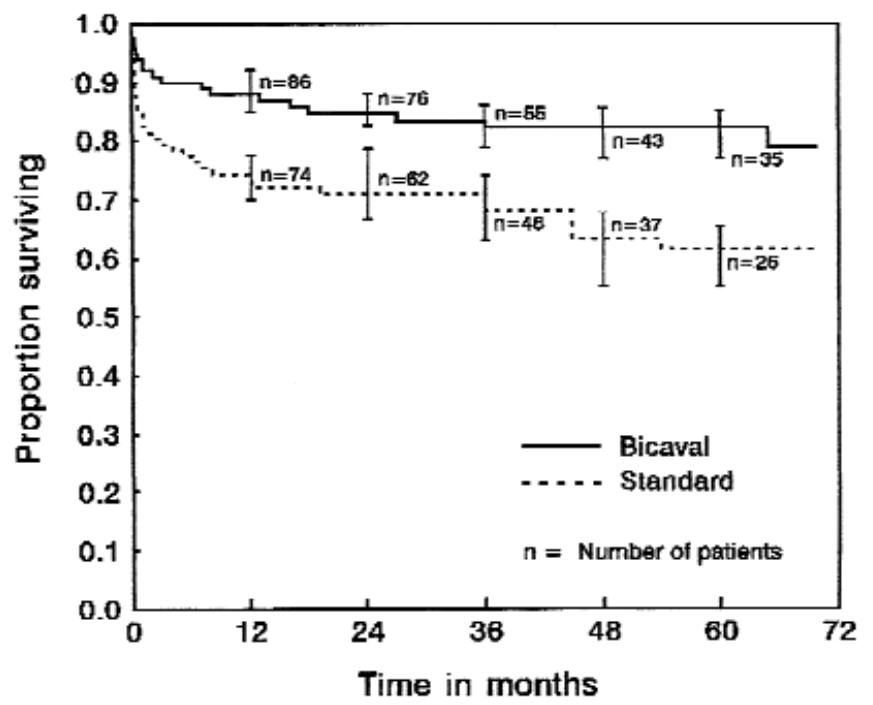

Fig 3. Actuarial survival between recipients of standard and bicaval heart transplants.

sure was also lower for the recipients of the bicaval technique $(P=.03)$.

Transthoracic echocardiogram was performed for each surviving recipient, and it revealed improved mean ejection fraction for the bicaval group $(P=.004)$.

Tricuspid valve regurgitation was observed in $80 \%$ (70/87 patients) in the standard versus $63 \%(57 / 91$ patients) in recipients of the bicaval technique $(P=$ .008). The prevalence of moderate to severe tricuspid valve dysfunction was higher for the standard technique $(31 / 87$ patients [35\%]) versus those patients who underwent the bicaval technique (19/91 patients [21\%]; $P=.02)$. The incidence of rejection was similar in the 2 groups (71 patients from the standard group and 76 patients from the bicaval group $[P=.9])$.

One- and two-year periods. Twelve months after the transplantation, the intracardiac pressures (RA and mean pulmonary pressures) were significantly lower for the recipients of the bicaval technique (Table III). The pulmonary wedge pressure was lower in the recipients of the bicaval technique $(P=.04)$.

The left ventricular ejection fraction was also better for this group $(P=.02)$ (Fig 1).

Thirty-five percent of recipients (27/78 cases) in the standard technique had moderate to severe tricuspid valve dysfunction versus $17 \%$ (15/87 cases) of the bicaval group $(P=.015$; Fig 2$)$.

Analysis of the data with respect to era revealed constantly lower RA and pulmonary pressures after 1 year for the recipients of the bicaval technique than for recipients of the standard technique in both eras. For the recipients of the bicaval technique, the RA pressure was
Table IV. Causes of death at 60 months after transplantation in the recipients of the standard and bicaval techniques

\begin{tabular}{lccc}
\hline Cause of death & $\begin{array}{c}\text { Standard } \\
(n=105)\end{array}$ & $\begin{array}{c}\text { Bicaval } \\
(n=96)\end{array}$ & $\mathrm{P}$ value \\
\hline Hospital & 18 & 6 & .03 \\
Cardiac & 13 & 3 & .01 \\
Infection & 2 & 3 & .6 \\
Cancer & 2 & 2 & .9 \\
Renal failure & 2 & 2 & .9 \\
Others & 2 & 3 & .6 \\
\hline
\end{tabular}

$4.03 \pm 4.1 \mathrm{~mm} \mathrm{Hg}$ during era 1 and $4.6 \pm 4.0 \mathrm{~mm} \mathrm{Hg}$ for era 2 versus $10.7 \pm 4.1$ for era 1 and $11.1 \pm 3.9 \mathrm{~mm} \mathrm{Hg}$ during era 2 for the recipients of the standard technique $(P=.002$ and .001 , respectively, for differences between techniques during era 1 and era 2). Mean PA pressure for the bicaval group was $17.6 \pm 4.3 \mathrm{~mm} \mathrm{Hg}$ during era 1 and $17.4 \pm 5.8 \mathrm{~mm} \mathrm{Hg}$ during era 2 compared with $22.8 \pm 5.1 \mathrm{~mm} \mathrm{Hg}$ and $21.9 \pm 5.8 \mathrm{~mm} \mathrm{Hg}$ during era 1 and era 2, respectively, for the recipients of the standard technique ( $P=.01$ and .008 , respectively).

At 2 years after transplantation, the mean left ventricular ejection fraction was better preserved for the recipients of the bicaval technique than for the recipients of the standard technique $(P=.014)$. Thirty-two percent of recipients (22/69 cases) with the standard technique showed significant (moderate or severe) tricuspid valve dysfunction versus $16 \%$ (13/80 cases) in the recipients of the bicaval technique $(P=.031$; Fig 2$)$.

Recent follow-up. After a mean follow-up period of 
$39 \pm 21$ months for the standard technique and $46 \pm 19$ months for the bicaval technique, the echocardiographic study recorded persistent improved mean left ventricular ejection fraction for the recipients of the bicaval technique $(P=.005)$. Recipients with the bicaval technique have shown lower incidence and severity of tricuspid valve dysfunction after orthotopic heart transplantation. Moderate or severe tricuspid valve dysfunction was recorded in $7 \%$ of the patients who underwent the bicaval versus $28 \%$ of recipients who underwent the standard technique of heart transplantation $(P=.018)$. The incidence of tricuspid valve dysfunction was higher in recipients of the standard technique, regardless of the era of transplantation. Moderate or severe tricuspid valve dysfunction was recorded in $30 \%$ and $26 \%$ of the recipients of the standard technique compared with $9 \%$ and $6.5 \%$ in the recipients of the bicaval technique performed during era 1 and era 2 , respectively $(P=.02$ and .016 , respectively). There was no difference between the incidence and severity of rejection between the 2 groups at any stage after the transplantation. The incidence of rejection episodes was $4.2 \pm 0.6$ for the standard group and $4.1 \pm 0.7$ for the bicaval group $(P=.9)$.

Survival. The actuarial survival at 1,3 , and 5 years was $74 \%, 70 \%$, and $62 \%$ for standard technique versus $87 \%, 82 \%$, and $80 \%$ for the bicaval technique $(P=$ $.028, .033$, and .018 , respectively). Death from cardiac causes was significantly higher for the recipients of the standard technique (Table IV; Fig 3). Analysis of survival per era revealed generally improved operative survival for both techniques during era 2 . However, there was a persistence of marginally better results for the recipients of the bicaval technique. During era 1, operative mortality was 4 of 41 patients (11\%) for recipients of the bicaval technique compared with 7 of 38 patients $(18 \%)$ for the recipients of the standard technique $(P=$ .3). In era 2 , operative mortality for the recipients of the bicaval technique was 2 of 55 patients (3.5\%) compared with 11 of 67 patients (16\%) for recipients of the standard technique performed during the same era $(P=$ .041). Persistent right-sided failure was responsible for most operative deaths in both techniques ( $5 / 6$ patients for the bicaval technique; $14 / 18$ patients for the standard technique).

\section{Discussion}

Results from our study illustrate the early and longterm influence of the surgical technique on right-sided hemodynamics, left ventricular function, and tricuspid valve function after heart transplantation. Immediately after heart transplantation in most patients, the right ventricle is exposed to elevated pulmonary pressure with the risk of acute right ventricular failure because of the afterload mismatch. Thus surgical technique leaving the right atrium intact with subsequent preservation of the RA function to enhance net stroke volume may have a positive effect on right ventricular function. Normal atrial pump function contributes up to $20 \%$ of the stroke volume. ${ }^{10,11}$ Because asynchronous contraction of recipient and donor atria and abnormal atrial size is a common finding after standard transplantation, atrial contribution to stroke volume might be diminished. ${ }^{4}$ In contrast to the standard technique, the bicaval technique offers a more physiologic RA size ${ }^{12}$ and contraction pattern throughout the cardiac cycle. ${ }^{13}$

The difference in the physiologic condition on the right side between the 2 techniques during the early postoperative period has been previously investigated. Diminished forward systolic flow in the superior vena cava has been demonstrated in patients undergoing transplantation by the standard technique, with similar systolic forward and diastolic flows for recipients of the bicaval technique. ${ }^{14}$ Diminished forward systolic vena caval flow has also been described in restrictive ventricular pathologic findings such as cardiomyopathy ${ }^{15}$ and acute cardiac rejection, and this pattern of abolished forward systolic vena caval flow has been proposed as a sign of acute cardiac rejection. ${ }^{16}$ The difference between the 2 techniques with respect to vena caval forward flow is likely to be related to the differences in RA size and performance. An increased late diastolic tricuspid flow in the patient with the bicaval technique indicated more vigorous RA contraction followed by better atrial relaxation, with increased forward vena caval flow during subsequent ventricular systole. ${ }^{17}$ In contrast, RA contraction may be less vigorous in recipients of the standard technique as a result of atrial sutures and greater size.

Generally, tricuspid valve dysfunction is reported to be highly prevalent both immediately and late after orthotopic heart transplantation..$^{4-18,19}$ Theories to explain this include acute allograft edema (which diminishes with time), ${ }^{20}$ papillary muscle dysfunction, ${ }^{21}$ preoperative annular dilatation, ${ }^{22}$ disturbed geometry of the RA anastomosis with subsequent impairment of the functional integrity of the valvular apparatus, ${ }^{18}$ possibly cyclic torsion of the atria during ventricular systole and diastole, ${ }^{18}$ asynchronous contraction of the donor and recipient atrial compartment, ${ }^{20}$ and biopsy-induced flail tricuspid valve. ${ }^{23}$ Alteration in the right ventricular structure may also contribute to the occurrence of tricuspid valve dysfunction because a significantly greater percentage of patients with post-transplantation right ventricular enlargement have been found to have moderate tricuspid 
valve dysfunction. ${ }^{24}$ Using the bicaval Wythenshawe technique, we have noted a marked reduction in early and late postoperative significant (moderate or severe) tricuspid valve dysfunction compared with patients undergoing transplantation with the standard technique. Of note is the fact that $50 \%$ of recipients with a severe degree of tricuspid valve dysfunction in our series (all from the group undergoing standard transplantation) had symptoms related to chronic right ventricular volume overload and their right-sided pressures were significantly elevated at the end of the first year after the transplantation.

Right and left ventricular performance after transplantation are affected by the surgical technique, and depressed right ventricular function with the standard technique has been documented as the result of significantly increased right ventricular volume and distortion of the right ventricular free wall relative to the interventricular septum. ${ }^{25}$ The echocardiographic analysis of cardiac transplant recipients in whom standard technique was used showed an enlarged right ventricular cavity and dilatation caused by distortion of the right ventricular free wall relative to the interventricular septum was shown by a significant increase in the septum-to-right ventricular free wall dimensions. ${ }^{25}$

The improvement in the right-sided hemodynamics for the recipients of the bicaval technique reflects improvement in their right ventricular function for this group of patients. Several reasons could explain this: improved atrial geometry with reduction in the incidence of tricuspid valve $e^{12,26-28}$ and improved right ventricular dimensions (right ventricular end-diastolic area) have been also reported in transplant recipients with the bicaval technique. ${ }^{29}$ Superior right and left ventricular diastolic function ${ }^{30}$ in recipients with bicaval technique contribute to improved right ventricular and mean PA compliance in the recipients of the bicaval technique.

The improvement of the left-sided performance during the early postoperative period after heart transplantation has also been reported ${ }^{6,7}$ after the use of the bicaval technique. Reduced left and right ventricular isovolumic relaxation times in patients who underwent transplantation by the bicaval technique probably indicate better relaxation, ${ }^{14}$ because the RA and the left atrial pressures were lower in these groups. Previous studies have reported that a small left atrial cuff with the bicaval technique lowered the incidence of mitral valve dysfunction and maintained the atrial contribution to the left ventricular filling. ${ }^{6-8}$

Potentially, the differences in survival in our study could be attributed to era of transplantation. The International Society for Heart and Lung Trans-
Table V. Analysis of first-year confounding factors in recipients of both standard and bicaval techniques (renal impairment serum creatinine of more than 200 $\mathrm{mmol} / \mathrm{L}$ for 4 weeks)

\begin{tabular}{lccc}
\hline & $\begin{array}{c}\text { Standard } \\
\text { technique } \\
(n=105)\end{array}$ & $\begin{array}{c}\text { Bicaval } \\
\text { technique } \\
(n=96)\end{array}$ & P value \\
Confounding factor & 89 & 90 & .9 \\
\hline Cellular rejection (n) & 2 & 3 & .9 \\
Humoral rejection (n) & & & .7 \\
Infection episode/patient & $0.29 \pm 0.25$ & $0.24 \pm 0.19$ & .8 \\
$\quad$ Bacterial & $0.11 \pm 0.03$ & $0.12 \pm 0.04$ & .8 \\
Viral & 0.006 & 0.005 & .8 \\
$\quad$ Fungal & $163 \pm 25$ & $167 \pm 21$ & .9 \\
Cyclosporine level & & & 3 \\
$\quad$ (mmol/L) & 4 & & \\
Renal impairment (n) & & & \\
\hline
\end{tabular}

plantation Official Registry ${ }^{31}$ considers that the period of 1991 through 1997 is involved in the actual current practice of transplantation. Half-life after transplantation was 9.2 years for 1991 compared with 9.4 years for 1997. Analysis of our data at 2 different stages of the current era (April 1991-April 1994 as era 1; May 1994-April 1997 as era 2) has demonstrated persistent superior cardiac function and lower incidence of tricuspid valve dysfunction in the recipients of the bicaval technique, which could be the reason behind the improved survival for the recipients of the bicaval technique.

Although the preoperative clinical characteristics that may have influenced the early postoperative outcome were not significantly different between the 2 groups (Table II), a limitation of our study was that that outcome could have been affected by postoperative clinical features. We did not, however, identify a difference between the 2 groups in terms of incidence or severity of rejection episodes, renal dysfunction, or infection, which are likely to be the major predictors of outcome after the operation (Table V). Therefore the difference in clinical outcome between the bicaval and standard groups is more likely to be attributable to the operative technique rather than any other factor.

Several studies have investigated the advantage of the bicaval technique during the early postoperative period after orthotopic heart transplantation. Our report illustrates the medium-term benefits of the bicaval technique in heart transplantation. Both right- and leftsided cardiac function were preserved for more than 5 years after the transplantation with less incidence of atrioventricular valve regurgitation. Death from cardiac reasons was lower in the bicaval group. 
This study highlights the importance of preference of the bicaval technique for orthotopic heart transplantation, especially in select young patients and, in particular, those potential transplant recipients with end-stage cardiac disease.

\section{REFERENCES}

1. Sarsam MA, Campbell CS, Yonan NA, Deiraniya AK, Rahman AN. An alternative technique in orthotopic cardiac transplantation. J Card Surg 1993;8:344-9.

2. Lower RR, Stofer RC, Shumway NE. Homovital transplantation of the heart. J Thorac Cardiovasc Surg 1961;41:196-201.

3. Shumway NE, Lower R, Stofer RC. Transplantation of the heart. Adv Surg 1966;2:265-84.

4. Angerman CE, Spes CH, Tammen A, et al. Anatomic characteristics and valvular function of the transplanted heart: transthoracic versus tranesophageal echocardiographic findings. J Heart Transplant 1990;9:331-8.

5. Jacquet L, Ziady G, Stein K, et al. Cardiac rhythm disturbance early after orthotopic heart transplantation: prevalence and clinical importance of observed abnormality. J Am Coll Cardiol 1990; 16:832-7.

6. El-Gamel A, Yonan NA, Grant S, Deiraniya AK, Rahman AN, Campbell CS. Orthotopic heart transplantation: a comparison between the standard and the bicaval Wythenshawe technique. J Thorac Cardiovasc Surg 1995;109:721-30.

7. El-Gamel A, Deiraniya AK, Rahman AN, Campbell CS, Yonan NA. Orthotopic heart transplantation hemodynamics: Does atrial preservation improve cardiac output after transplantation? J Heart Lung Transplant 1996;15:564-71.

8. Deleuze PH, Benvenuti C, Mazzuotelli JP, et al. Orthotopic cardiac transplantation with direct caval anastomosis: Is it the optimal procedure? J Thorac Cardiovasc Surg 1995;109:731-73.

10. Midei MG, Baugham KL, Achuff SC, Walford GD, Baumgartner W, Brinker JA. Is atrial activation beneficial in heart transplant recipients? J Am Coll Cardiol 1990;36:1201-4.

11. Goldstein JA, Barzali B, Rosamond TL, Eisenberg PR, Jaffe AS. Determination of hemodynamic compromise with severe right ventricular infarction. Circulation 1990;82:359-68.

12. Sievers H-H, Leyh R, Jahank A, et al. Bicaval versus atrial anastomoses in cardiac transplantation. J Thorac Cardiovasc Surg 1994;108:708-4.

13. Bizouran P, Treilhaud M, Portier D, Train M, Michaud J-L. Right ventricular function early after total or standard orthotopic heart transplant. Ann Thorac Surg 1994;57:183-7.

14. Peteiro J, Redondo F, Cuenca J, Pradas G, Beirac AC. Difference in heart transplant physiology according to surgical technique. J Thorac Cardiovasc 1996;112:584-9.

15. Hatle LK, Appleton CP, Popp RL, Difference in constrictive pericarditis and restrictive cardiomyopathy by Doppler echocardiography. Circulation 1989;79:357-70.

16. Simmonds MB, Lythall DA, Slorach C, Ilsy CD, Mitchell AG, Yacoub MH. Doppler examination of superior vena caval flow for the detection of acute cardiac rejection. Circulation 1992;86 (Suppl):259-66.

17. Nishimura RA, Abel MD, Hatle LK, Tajik AJ. Relationship of pulmonary vein to mitral velocities by transesophageal Doppler echocardiography: effect of different loading conditions. Circulation 1990;81:1488-97.

18. Stevenson LW, Dadourian BJ, Kobashigawa J, Child JS, Clark SH, Laks H. Mitral regurgitation after cardiac transplantation. Am J Cardiol 1987;60:119-22.

19. Lewen MK, Bryk RJ, Miller LW, Williams GA, Labowtiz AJ. Tricuspid regurgitation by Doppler echocardiography after orthotopic cardiac transplantation. Am J Cardiol 1987;59:1371-4.

20. Cladellas M, Abdal ML, Pons-Llado G, et al. Early transient multivalvular regurgitation detected by pulsed Doppler in cardiac transplantation. Am J Cardiol 1986;58:1122-4.

21. Akasaka T, Lythall DA, Kushawaha SS, Yoshida K, Yoshikawa J, Yacoub MH. Valvular regurgitation in heart-lung transplant recipients: a Doppler color flow study. J Am Coll Cardiol 1990;15: 576-81.

22. Rees AP, Milani RV, Lavie CJ, Smart FW, Ventura HO. Valvular regurgitation and right-side cardiac pressures in heart transplant recipients by complete Doppler and color flow evaluation. Chest 1993;104:82-7.

23. Williams MAJ, Lee MY, Di Salvo TG, et al. Biopsy-induced flail tricuspid leaflets and tricuspid regurgitation following orthotopic cardiac transplantation. Am J Cardiol 1996;77:1339-44.

24. Bhatia SJS, Kirshenbaum JM, Shemin RJ, et al. Time course of resolution of pulmonary hypertension after orthotopic cardiac transplantation. Circulation 1987;76:819-26.

25. Bittner HB, Kendale SW, Chen EP, Davis RD, Tright PV III Complete atrioventricular transplantation: improved performance compared with the standard technique. Ann Thorac Surg 1995; 60:275-83.

26. Beniaminovitz A, Savoia MT, Galantowicz M, Di-Tullio MR, Homma S, Mancini D. Improved atrial function in bicaval versus standard orthotopic techniques in cardiac transplantation. Am J Cardiol 1997;80:1631-5.

27. Forni A, Faggian G, Chiominto B, et al. Avoidance of atrioventricular valve incompetence following orthotopic heart transplantation using direct bicaval anastomosis. Transplant Proc 1995; 27:3478-82.

28. Laske A, Carrel T, Niederhauser U, et al. Modified operation technique for orthotopic heart transplantation. Eur J Cardiothorac Surg 1995;9:120-6.

29. Leyh RG, Jahnke AW, Kraatz EG, Sievers HH. Cardiovascular dynamics and dimensions after bicaval and standard cardiac transplantation. Ann Thorac Surg 1995;59:1495-500.

30. El Gamel A, Yonan NA, Keevil B, Warbuton R, Kakadellis J, Woodcock A, Campbell CS, et al. Significant of raised natriuretic peptides after bicaval and standard cardiac transplantation. Ann Thorac Surg 1997;63:1095-100.

31. Hospend JD, Bennett LE, Keck BM, Fiol B, Boucek MM, Novick RJ. The Registry of the International Society for Heart and Lung Transplantation. J Heart Lung Transplant 1998;17:665-8. 